\title{
Carpometacarpal Joint 1
}

National Cancer Institute

\section{Source}

National Cancer Institute. CarpometacarpalJoint 1. NCI Thesaurus. Code C103912.

A saddle-shaped synovial joint between the metacarpal of the thumb and the trapezium. 